\title{
Maternal Thyroid Disease as a Risk Factor for Craniosynostosis
}

\author{
Sonja A. Rasmussen, MD, MS, Mahsa M. Yazdy, MPH, Suzan L. Carmichael, PhD, \\ Denise J. Jamieson, MD, MPH, Mark A. Canfield, PhD, and Margaret A. Honein, PhD, MPH, \\ for the National Birth Defects Prevention Study
}

OBJECTIVE: To study the relationship between maternal thyroid disease and craniosynostosis using data from the National Birth Defects Prevention Study, a multisite, case-control study.

METHODS: Case infants $(n=431)$ were identified through population-based birth defects surveillance systems at eight sites and had craniosynostosis verified by radiographic imaging. Control infants $(n=4,094)$ consisted of a random sample of live births with no major birth defects from the same population as the case

From the National Center on Birth Defects and Developmental Disabilities, Centers for Disease Control and Prevention, Atlanta, Georgia; Oak Ridge Institute for Science and Education (ORISE) Fellowship Program, Oak Ridge, Tennessee; March of Dimes Birth Defect Foundation/California Birth Defects Monitoring Program, California Department of Health Services, Berkeley, California; National Center for Chronic Disease Prevention and Health Promotion, Centers for Disease Control and Prevention, Atlanta, Georgia; and Birth Defects Epidemiology and Surveillance Branch, Texas Department of State Health Services, Austin, Texas.

Funded by the Centers for Disease Control and Prevention.

The authors thank the participating families and study investigators and staff at the National Birth Defects Prevention Study sites for their important contributions. The authors also thank Sarah Collier, MPH, of the Centers for Disease Control and Prevention for her assistance with replicating the analysis of these data. Coding of drug information in the National Birth Defects Prevention Study used the Slone Drug Dictionary, under license from the Slone Epidemiology Center at Boston University.

Presented at the 2006 Teratology Society annual meeting, Tucson, Arizona, June 24-29, 2006, and the 2006 David W. Smith Workshop on Malformations and Morphogenesis, Lake Arrowhead, California, September 8-13, 2006.

The findings and conclusions in this report are those of the authors and do not necessarily represent the viewes of the Centers for Disease Control and Prevention.

Use of trade names and commercial sources does not imply endorsement by the Centers for Disease Control and Prevention of the U.S. Department of Health and Human Services.

Corresponding author: Sonja A. Rasmussen, MD, MS, 1600 Clifton Road, MS E-86, Centers for Disease Control and Prevention, Atlanta, GA 30333; e-mail: skr9@cdc.gov.

\section{Financial Disclosure}

The authors have no potential conflicts of interest to disclose.

(C) 2007 by The American College of Obstetricians and Gynecologists. Published by Lippincott Williams \& Wilkins.

ISSN: 0029-7844/07 infants. Information on thyroid disease was based on self-report: mothers who reported either a thyroid disorder or use of a medication to treat a thyroid disorder during pregnancy were considered to have thyroid disease. Using an unconditional logistic regression model, we considered potential confounding factors (maternal age, race or ethnicity, smoking, body mass index, preexisting diabetes, plurality, gravidity, family history, infant sex).

RESULTS: Among case mothers, 19 (4.4\%) were classified as having thyroid disease, compared with 65 (1.6\%) of control mothers. Maternal thyroid disease was associated with craniosynostosis after controlling for maternal age (adjusted odds ratio 2.47, 95\% confidence interval 1.46$4.18)$, the only factor that remained significant in the final model.

CONCLUSION: These data provide additional evidence that maternal thyroid disease (most likely Graves' disease) or its treatment is associated with craniosynostosis. Given the frequency of maternal thyroid disease, this association warrants further investigation.

(Obstet Gynecol 2007;110:369-77)

\section{LEVEL OF EVIDENCE: II}

B ecause thyroid disease is the second most common endocrinopathy affecting reproductive-aged women, ${ }^{1}$ management of thyroid disease in pregnancy is relatively common in obstetric practice. ${ }^{2}$ Overt hypothyroidism affects $0.3-0.7 \%$ of women of reproductive age, whereas subclinical hypothyroidism affects 2.5\% ${ }^{3,4}$ Hyperthyroidism, most often due to Graves' disease, occurs in about $0.2 \%$ of pregnancies. ${ }^{4,5}$ Pregnancies affected by either hypothyroidism or hyperthyroidism have been associated with adverse outcomes. ${ }^{4-8}$ In addition, a significant proportion of pregnancies $(0.5-1 \%)$ occur among women with a past history of Graves' disease, and these women are also at risk for adverse pregnancy outcomes; even after surgical treatment or radioactive 
ablation of the thyroid, women can continue to produce thyroid-stimulating immunoglobulins that cross the placenta, with potential adverse fetal effects. ${ }^{9}$

Craniosynostosis is a birth defect characterized by premature fusion of one or more cranial sutures. The defect occurs in approximately one in 2,000 births, ${ }^{10}$ and usually requires surgical correction. Untreated, craniosynostosis can lead to an abnormal head shape, restriction of brain growth, and increased intracranial pressure. Premature suture closure occurs prenatally or perinatally, but also can occur later during infancy. Genetic and environmental causes of craniosynostosis have been identified, but in most cases the cause is unknown. ${ }^{11,12}$ Craniosynostosis has been observed among children with postnatal Graves' disease or hyperthyroidism ${ }^{13-19}$ and with excess thyroid hormone treatment during childhood. ${ }^{14,20}$ In addition, review of the literature reveals several infants with craniosynostosis born to mothers with active or a past history of maternal Graves' disease (Leonard CO, Ralston C, Carey JC, Morales L. Craniosynostosis and facial dysmorphism due to maternal Graves disease [abstract]. Clin Res 1987;35: 225A). ${ }^{21-25}$ These case reports suggest that this birth defect could sometimes result from increased thyroid hormone at a critical period during fetal or early infant growth.

Given the frequency of thyroid disease among reproductive-aged women and the reports suggesting an association between excess thyroid hormone and craniosynostosis, we examined the relation between maternal thyroid disease and craniosynostosis using data from the National Birth Defects Prevention Study, a multisite case-control study of major birth defects.

\section{MATERIALS AND METHODS}

The National Birth Defects Prevention Study is an ongoing, population-based, case-control study of genetic and environmental risk factors for major birth defects. Detailed study methods have been published elsewhere ${ }^{26}$ Case infants are ascertained from birth defects surveillance systems at eight sites (Arkansas, California, Georgia, Iowa, Massachusetts, New Jersey, New York, and Texas) with at least one of more than 30 study-eligible birth defects. The study was approved by institutional review boards at all study sites. Because the focus of the National Birth Defects Prevention Study is on identifying risk factors for birth defects of unknown cause, infants known or strongly suspected to have a condition of known cause (chromosome abnormality or single-gene condition) are excluded from the study. Controls were liveborn infants without birth defects randomly selected from birth certificates or birth hospital records from the same source population and time period as the cases. The source population was defined based on maternal residence at the time of delivery. Mothers of case and control infants complete a telephone interview from 6 weeks through 24 months after their estimated date of delivery. Mothers are asked questions on a wide variety of topics including maternal illnesses, pregnancy history, vitamin intake, tobacco use, alcohol intake, substance abuse, environmental and occupational exposures, and medication history. Information on case infants is reviewed by a clinical geneticist at each site before inclusion to ensure that case definitions for the study are met. ${ }^{27}$

The present analysis is limited to study of the defect, craniosynostosis. For inclusion in the study, craniosynostosis must have been verified by radiographic imaging (eg, skull radiograph or head computed tomography scan). Based on the radiographic imaging studies, craniosynostosis was classified by type of major suture involved (ie, sagittal, coronal, metopic, lambdoidal, multiple, or unknown). Infants with more than one type of suture involved (eg, sagittal and coronal sutures) were counted only in the multiple suture category. This analysis was limited to case and control infants born on or after October 1, 1997, and with an estimated date of delivery on or before December 31, 2002, whose mothers completed the telephone interview.

The exposure of interest was defined as maternal thyroid disease. A mother was considered to have thyroid disease if she reported either having a thyroid disorder or taking thyroid medication any time during pregnancy. In the study questionnaire, mothers were asked whether they had "any other diseases or illnesses that [they] haven't already talked about, such as chronic diseases, infectious diseases, or sexually transmitted diseases." The responses to this question, as well as the interviewer comment fields, were searched for any mention of a thyroid disorder (eg, hypothyroidism, Hashimoto's thyroiditis, hyperactive thyroid, disorder of the thyroid, thyroiditis, hyperthyroidism, or Graves' disease). Mothers were asked whether they took any of a specific list of medications, and then asked about use of any other medications during the period 3 months before through the end of pregnancy; the name of the medications and timing of use were documented. The following list of medications was used to identify mothers treated for a thyroid disease: liothyronine, liothyronine sodium, liothyronine $\mathrm{HCl}$, levothyroxine sodium (Synthroid [Knoll Pharmaceuticals, Mt. Olive, NJ], Levothroid 
[Forest Pharmaceutical, Inc., St. Louis, MO], and Levoxyl [Jones Medical Industries, Inc., St. Louis, $\mathrm{MO}]$ ), propylthiouracil, methimazole, carbimazole, thyroid hormone, thyroglobulin, potassium perchlorate, thyrotrophin, thyroxine, thyroid tissue, thyroxine I 125, and thyroid medication not otherwise specified. A clinician (S.A.R.) who was blinded to case-control status reviewed the interview responses of mothers identified as possibly having thyroid disease to confirm that they met our exposure definition. Medication timing and pattern of use for mothers taking any thyroid medication were examined. We also reviewed information from the maternal interview regarding the type of thyroid disorder and specific thyroid medications used by case and control mothers who had been classified as having thyroid disease.

When case infants are ascertained by birth defects surveillance systems, information on maternal conditions and medications taken prenatally is sometimes abstracted and transferred to the National Birth Defects Prevention Study clinical database, in addition to information on birth defects. We reviewed information from the study clinical database or, when available, from additional review of medical records on case infants whose mothers had reported a thyroid disorder or thyroid medication. This information was used to better inform the possible mechanism involved in the association between thyroid disease and craniosynostosis, but was not incorporated into our analysis, given that this information was not available for control infants.

Statistical analyses were conducted using Statistical Analysis Battery for Epidemiological Research (SABER v1.96; Centers for Disease Control and Prevention) and SAS 9.1 software (SAS Institute, Cary, NC). To control for potential confounding and calculate adjusted odds ratios (ORs) and 95\% confidence intervals (CIs), we fit an unconditional logistic regression model. Our initial full model included all potential risk factors for craniosynostosis assessed in the univariable analyses: maternal race or ethnicity (non-Hispanic white, non-Hispanic African American, Hispanic, Asian or Pacific Islander, Native American, other), periconceptional maternal smoking (any use from one month before pregnancy through the end of the first trimester, no smoking), infant sex (male, female), family history of craniosynostosis in a first-degree relative (yes, no), maternal age (19 years or younger, $20-29,30-39,40$ years or older), pregestational maternal diabetes (yes, no), prepregnancy body mass index (BMI) (BMI less than 18.5, BMI 18.5-24.9, BMI 25-29.9, BMI 30 or more), maternal education (less than 12 years, 12 years, more than 12 years), plurality (singleton, other), and gravidity (primigravid, multigravid). Each variable was removed from the model one at a time. If removing the variable resulted in a change in the effect estimate (OR) of more than $10 \%$, it was placed back in the model. The final model only included maternal age in the four categories listed previously. After excluding infants with a family history of craniosynostosis in a first-degree relative and mothers with pregestational diabetes, analyses were repeated. Stratified analyses by study site and by type of cranial suture prematurely fused were performed. We also examined those case infants with isolated craniosynostosis (those with no other unrelated major birth defects) separately. ${ }^{27}$

\section{RESULTS}

Participation rates for the time period of this analysis were $69 \%$ for mothers of control infants and $73 \%$ for mothers of infants with craniosynostosis. A total of 431 case infants with craniosynostosis and 4,094 control infants with completed maternal interviews were available for analysis. The most frequent suture involved among infants with craniosynostosis was the sagittal suture, involved in $212(49.2 \%)$ of case infants (Table 1). Most infants with craniosynostosis (88.9\%) had a single type of suture involved and most (89.8\%) were classified as having isolated craniosynostosis (no other major, unrelated defects).

Infants with craniosynostosis were more likely than control infants to be male, born at preterm gestation, a product of a twin gestation or higher, and to have a birth weight that was either low (less than

\section{Table 1. Types of Craniosynostosis Among Case Infants, National Birth Defects Prevention Study, 1997-2002}

\begin{tabular}{lc}
\hline Characteristics & Number (\%) \\
\hline Type of suture involved & \\
Coronal & $22(19.0)$ \\
Lambdoidal & $21(4.9)$ \\
Metopic & $68(15.8)$ \\
Sagittal & $212(49.2)$ \\
Unknown & $2(0.5)$ \\
Multiple & $46(10.7)$ \\
Number of suture types affected & \\
One & $383(88.9)$ \\
Two & $39(9.0)$ \\
Three or more & $7(1.6)$ \\
Unknown & $2(0.5)$ \\
Infant classification & \\
Isolated* & $387(89.8)$ \\
Multiple & $44(10.2)$ \\
\hline * Infants with no other unrelated major birth defects. & \\
$\dagger$ Infants with other unrelated major birth defects. &
\end{tabular}


2,500 g) or macrosomic (4,000 g or more) (Table 2). Mothers of infants with craniosynostosis were more likely than mothers of control infants to be older, have a higher level of education, be of non-Hispanic white ethnicity, have a BMI in the obese range, to be multigravid, and to have pregestational diabetes. Among mothers of infants with craniosynostosis, $4.4 \%$ $(n=19)$ reported thyroid disease or its treatment, compared with only $1.6 \% \quad(n=65)$ of mothers of control infants (crude OR 2.86, 95\% CI 1.64-4.94).

After considering all previously described potential confounders, only maternal age was significant and therefore included in the final model. The association between maternal thyroid disease and craniosynostosis remained after controlling for maternal age (adjusted OR 2.47, 95\% CI 1.46-4.18). Results were similar after excluding infants with a first-degree relative with craniosynostosis and infants whose mothers had pregestational diabetes (data not shown). Stratification by study site showed similar associations across all sites and confirmed that no single study site was responsible for the observed association. The association seemed to vary by the type of suture involved. Odds ratios were elevated for sagittal and coronal craniosynostosis (adjusted ORs $2.11,95 \%$ CI $0.99-4.48$ and $3.32,95 \%$ CI $1.29-8.52$, respectively), and the association was strongest for infants with involvement of more than one suture (adjusted OR 8.73, 95\% CI 3.54-21.57). Odds ratios for metopic and lambdoid craniosynostosis were not calculated because no mothers of case infants with only metopic or lambdoid suture involvement reported maternal thyroid disease. Although nearly $16 \%$ of infants whose mothers did not have maternal thyroid disease had metopic suture involvement, none of the infants whose mothers had maternal thyroid disease had metopic suture involvement. When we examined data on infants classified as having isolated craniosynostosis (those with no other major, unrelated birth defects), the odds ratio remained elevated (adjusted OR 2.62, 95\% CI 1.53-4.48). The odds ratio for isolated craniosynostosis involving more than one suture type was particularly elevated (adjusted OR 9.56, 95\% CI 3.85-23.74).

Based on information from the maternal interview, most case mothers (73.7\%) classified as having thyroid disease reported a thyroid disorder, compared with less than half $(44.6 \%)$ of control mothers (Table 3). The most commonly reported condition was hypothyroidism for both case and control mothers (36.8\% and 18.5\% respectively). Graves' disease was reported in three of 19 (nearly 16\%) case mothers, compared with about $3 \%$ of control mothers classified as having thyroid disease. Hashimoto's thyroiditis was also more commonly reported by case mothers than control mothers $(10.5 \%$ compared with $1.5 \%$, respectively). All case mothers and most control mothers $(93.8 \%)$ who were classified as having thyroid disease reported taking a thyroid medication. Levothyroxine was the most commonly used medication among case and control mothers. Among mothers who reported taking any thyroid medication during pregnancy, $96.2 \%$ also had reported intake of a thyroid medication during the month before pregnancy. Among women who reported taking a thyroid medication during the month before pregnancy, nearly all $(97.4 \%)$ continued through the entire first and second trimesters of pregnancy.

Further information on maternal thyroid disease or its treatment was available from the study clinical database or from review of medical records on 16 case mothers classified as having thyroid disease. In nine, additional details beyond those supplied in the maternal interview were provided. Two of the three case mothers identified as having Graves' disease from maternal interview had been treated previously with radioiodine ablation of their thyroid glands. One case mother who reported Hashimoto's disease and levothyroxine treatment on maternal interview was noted to have had a thyroidectomy in the past. Another case mother, who reported hypothyroidism treated with levothyroxine in the maternal interview, was noted to have had Graves' disease, but was treated for hypothyroidism at the time of pregnancy. A case mother, who reported an unspecified thyroid disease with levothyroxine treatment on maternal interview, was noted to have a growth on her thyroid requiring a thyroidectomy before pregnancy. Another case mother with an unspecified thyroid disease with levothyroxine treatment on maternal interview was noted to have hypothyroidism. Of five case mothers noted to be on levothyroxine, but without report of thyroid disease on maternal interview, two were noted to have hypothyroidism, one had a previous partial thyroidectomy, and one had history of a thyroid cyst.

\section{DISCUSSION}

Mothers of infants with craniosynostosis were more than two times more likely to report thyroid disease or its treatment than mothers of control infants. This finding remained after adjustment for maternal age and assessment of other potential confounding factors. Our results are consistent with previous case reports of craniosynostosis after exposure to increased thyroid hormone levels, either prenatally ${ }^{21-25}$ or dur- 
Table 2. Descriptive Characteristics of Infants With Craniosynostosis and Infants With No Major Birth Defects, National Birth Defects Prevention Study, 1997-2002

\begin{tabular}{|c|c|c|c|}
\hline Characteristics & $\begin{array}{l}\text { Infants With Craniosynostosis } \\
\qquad(n=431)\end{array}$ & $\begin{array}{l}\text { Infants With no Major Birth Defects } \\
\qquad(\mathrm{n}=4,094)\end{array}$ & $P^{*}$ \\
\hline Infant sex & & & $<.001$ \\
\hline Male & $292(67.7)$ & $2,049(50.1)$ & \\
\hline Female & $139(32.3)$ & $2,040(49.8)$ & \\
\hline Missing & $0(0)$ & $5(0.1)$ & \\
\hline Maternal age (y) & & & $<.001$ \\
\hline 19 or younger & $28(6.5)$ & $459(11.2)$ & \\
\hline $20-29$ & $166(38.5)$ & $1,944(47.5)$ & \\
\hline $30-39$ & $214(49.7)$ & $1,598(39.0)$ & \\
\hline 40 or older & $23(5.3)$ & $93(2.3)$ & \\
\hline Maternal education (y) & & & .006 \\
\hline Less than 12 & $48(11.1)$ & $676(16.5)$ & \\
\hline 12 & $103(23.9)$ & $1,030(25.2)$ & \\
\hline More than 12 & $280(65.0)$ & $2,380(58.1)$ & \\
\hline Missing & $0(0)$ & $8(0.2)$ & \\
\hline Maternal race or ethnicity & & & $<.001$ \\
\hline Non-Hispanic white & $314(72.9)$ & $2,456(60.0)$ & \\
\hline Non-Hispanic African American & $19(4.4)$ & $491(12.0)$ & \\
\hline Hispanic & $79(18.3)$ & $931(22.7)$ & \\
\hline Asian or Pacific Islander & $9(2.1)$ & $122(3.0)$ & \\
\hline Native American or Alaskan native & $3(0.7)$ & $16(0.4)$ & \\
\hline Other & $7(1.6)$ & $67(1.6)$ & \\
\hline Missing & $0(0)$ & $11(0.3)$ & \\
\hline Maternal smoking & & & .57 \\
\hline Periconceptional smoking $^{\dagger}$ & $89(20.6)$ & $798(19.5)$ & \\
\hline No reported smoking & $342(79.4)$ & $3,295(80.5)$ & \\
\hline Missing & $0(0)$ & $1(0.02)$ & \\
\hline Maternal BMI (before pregnancy) & & & .31 \\
\hline Underweight (less than 18.5) & $22(5.1)$ & $233(5.7)$ & \\
\hline Normal weight $(18.5-24.9)$ & $227(52.7)$ & $2,254(55.1)$ & \\
\hline Overweight (25-29.9) & $105(24.4)$ & $862(21.1)$ & \\
\hline Obese (30 or more) & $71(16.5)$ & $584(14.3)$ & \\
\hline Missing & $6(1.4)$ & $161(3.9)$ & \\
\hline Pregestational maternal diabetes & & & .07 \\
\hline Yes & $5(1.2)$ & $20(0.5)$ & \\
\hline No & $425(98.6)$ & $4,067(99.3)$ & \\
\hline Missing & $1(0.2)$ & $7(0.2)$ & \\
\hline Gravidity & & & .10 \\
\hline Primigravid & $107(24.8)$ & $1,171(28.6)$ & \\
\hline Multigravid & $324(75.2)$ & $2,921(71.4)$ & \\
\hline Missing & $0(0)$ & $2(0.05)$ & \\
\hline Plurality & & & .002 \\
\hline Singleton & $405(94.0)$ & $3,959(96.7)$ & \\
\hline Twins or higher order & $26(6.0)$ & $130(3.2)$ & \\
\hline Missing & $0(0)$ & $5(0.1)$ & \\
\hline Gestational age & & & $<.001$ \\
\hline Preterm (less than 37 weeks) & $67(15.6)$ & $365(8.9)$ & \\
\hline Term (37 weeks or more) & $364(84.5)$ & $3,726(91.0)$ & \\
\hline Missing & $0(0)$ & $3(0.1)$ & \\
\hline Birth weight & & & $<.001$ \\
\hline Less than $1,500 \mathrm{~g}$ (very low) & $10(2.3)$ & $28(0.7)$ & \\
\hline $1,500-2,499 \mathrm{~g}$ (low) & $33(7.7)$ & $209(5.1)$ & \\
\hline $2,500-3,999 \mathrm{~g}$ (normal) & $316(73.3)$ & $3,405(83.2)$ & \\
\hline $4,000 \mathrm{~g}$ or more (macrosomic) & $57(13.2)$ & $431(10.5)$ & \\
\hline Missing & $15(3.5)$ & $21(0.5)$ & \\
\hline Maternal thyroid disease & & & $<.001$ \\
\hline Yes & $19(4.4)$ & $65(1.6)$ & \\
\hline No & $412(95.6)$ & $4,029(98.4)$ & \\
\hline
\end{tabular}

BMI, body mass index.

Data are $\mathrm{n}(\%)$.

* $P$ values calculated using $\chi^{2}$.

$\dagger$ Reported maternal smoking at any time from one month before pregnancy through the end of the first trimester.

VOL. 110, NO. 2, PART 1, AUGUST 2007 Rasmussen et al Maternal Thyroid Disease and Craniosynostosis 
Table 3. Reported Thyroid Disease and Medication Use (Based on Maternal Interview) Among Case and Control Mothers Classified as Having Thyroid Disease

\begin{tabular}{lcc}
\hline Thyroid Disease or Medication & Case Mothers $(\mathbf{n = 1 9 )}$ & Control Mothers $(\mathbf{n}=\mathbf{6 5})$ \\
\hline Report of thyroid disease* & $14(73.7)$ & $29(44.6)$ \\
Graves' disease & $3(15.8)$ & $2(3.1)$ \\
Hashimoto thyroiditis & $2(10.5)$ & $1(1.5)$ \\
Hypothyroid, low thyroid & $7(36.8)$ & $12(18.5)$ \\
Hyperthyroid & $0(0)$ & $2(3.1)$ \\
All other thyroid disease, specified & $0(0)$ & $3(4.6)$ \\
Thyroid disorder, not otherwise specified & $2(10.5)$ & $9(13.8)$ \\
No report of thyroid disease* & $5(26.3)$ & $36(55.4)$ \\
Report of thyroid medication ${ }^{\dagger}$ & $19(100.0)$ & $61(93.8)$ \\
Levothyroxine & $18(94.7)$ & $56(86.2)$ \\
Propylthiouracil & $1(5.3)$ & $4(6.2)$ \\
Methimazole & $1(5.3)$ & $3(4.6)$ \\
Other thyroid medication, specified & $0(0)$ & $0(0)$ \\
Thyroid medication, not otherwise specified & $0(0)$ & $2(3.1)$ \\
No report of thyroid medication & $0(0)$ & $4(6.2)$
\end{tabular}

Data are $\mathrm{n}(\%)$.

* Based on responses to question asking whether they had "any other diseases or illnesses that [they] haven't already talked about, such as chronic diseases, infectious diseases, or sexually transmitted diseases."

$\dagger$ Several mothers reported using more than one thyroid medication.

ing early infant development. ${ }^{13-20}$ Our results are also biologically plausible, given thyroid hormone's wellestablished role in skeletal development, ${ }^{28}$ and supported by animal studies. ${ }^{29}$ In contrast, infants with low thyroid hormone levels (congenital hypothyroidism) have late closure of the fontanelles, ${ }^{30}$ and most likely, less developed sutures. Our findings of varying effects by cranial suture type correspond with what is known about timing of closure of different suture types; metopic sutures are usually closed by age 3 years, compared with age of closure of other types of major cranial sutures (beginning in adulthood). ${ }^{11}$

Previous epidemiologic studies have not identified a statistically significant association between maternal thyroid disease and craniosynostosis, but these studies have been limited by small sample size ${ }^{31-33}$ and possibly problems with ascertainment of craniosynostosis, a defect usually not diagnosed at birth. ${ }^{31}$ However, in an epidemiologic study of maternal medication use and the risk for craniosynostosis, investigators excluded four of 212 infants with craniosynostosis and 1 of 291 controls who reported taking thyroid medications from their analysis ${ }^{34}$ because of the recognized association between thyroid medications and birth defects.

Several previous case reports of maternal Graves' disease and craniosynostosis have described mothers with active Graves' disease who were thyrotoxic during pregnancy ${ }^{21,23-25}$ and mothers with a history of Graves' disease who were euthyroid during pregnancy (Leonard et al [abstract]). ${ }^{21,22}$ These findings implicate maternal antibodies transmitted to the fetus with stimulation of the fetal thyroid to make excess amounts of thyroid hormone as the mechanism of craniosynostosis, rather than thyroxine produced by the mother or ingested from an exogenous source. In Graves' disease, thyroid-stimulating immunoglobulins (TSIs) (also referred to as thyroid-stimulating hormone [TSH]-receptor stimulating antibodies) and TSH binding inhibitory immunoglobulins bind to the TSH receptor to produce thyroid stimulation or inhibition, respectively. These antibodies freely cross the placenta and bind to the fetal thyroid to produce hyperthyroidism or hypothyroidism, whereas thyroid hormone crosses the placenta only in small amounts. ${ }^{9}$

In some ${ }^{21,23,24}$ but not all (Leonard et al [abstract $])^{25}$ of the previous reports with maternal thyroid disease and craniosynostosis, the infant has presented with manifestations of neonatal thyrotoxicosis (eg, tachycardia, irritability, hypertension, and poor weight gain). ${ }^{35}$ In nine infants with neonatal thyrotoxicosis born to mothers with active or a past history of Graves' disease, six of eight in whom skull radiographs were performed had craniosynostosis. ${ }^{21} \mathrm{Neo}-$ natal thyrotoxicosis is estimated to occur in $1-12 \%$ of neonates born to women with Graves' disease. ${ }^{35}$ Although much more rare, this condition has also been observed among infants born to women with Hashimoto's thyroiditis, who also can produce antibodies with thyroid-stimulating activity, ${ }^{36-38}$ and at least one of those infants developed craniosynostosis. ${ }^{36}$ Measurement of maternal TSIs has been shown to be a useful tool for predicting development of neonatal thyrotoxicosis. ${ }^{39}$ Based on knowledge of risk 
factors for neonatal thyrotoxicosis, ${ }^{9,22,40}$ endocrinologists have established guidelines for TSI screening, with measurement early in pregnancy for euthyroid women with previous radioiodine treatment or thyroid surgery for Graves' disease, and in the third trimester for women on antithyroid medications during pregnancy. ${ }^{9}$ Recommendations for follow-up of pregnancies with elevated TSIs for development of fetal or neonatal thyrotoxicosis $\operatorname{differ}^{41}$ and range from monitoring for fetal tachycardia, intrauterine growth restriction, or fetal thyroid gland size by ultrasonography, ${ }^{42}$ to monitoring of fetal thyroid function using umbilical blood sampling. ${ }^{43}$ Maternal treatment with antithyroid medications should be considered in fetuses with evidence of thyrotoxicosis. ${ }^{44}$ Routine measurement of TSI levels has not been recommended by the American College of Obstetricians and Gynecologists, although measurement is acknowledged to be of importance under some circumstances. ${ }^{1}$ Our finding of an association between maternal thyroid disease and craniosynostosis, which is most likely to be antibody-mediated among women with active or a past history of Graves' disease, might suggest another clinically important role for TSI testing in pregnancy.

Our results should be considered in the context of the study's limitations. Information on thyroid disease was based on mother's report and only limited information on the type of thyroid disease was available. However, the rate of maternal thyroid disease among controls identified by our study was similar to that observed in other studies of recognized thyroid disease, suggesting that women are accurately reporting the presence of overt maternal thyroid disease or its treatment. Additionally, information on thyroid disease or its treatment in case mothers obtained from maternal interview was supported by information abstracted from medical records in most cases. Another limitation is that recall bias (mothers of case infants might be more likely to recall exposures or maternal disease than control mothers) as a possible explanation for our findings cannot be excluded, given the retrospective study design. Our study showed that control mothers were less likely than case mothers to report thyroid disease, even when taking a medication for thyroid disease. However, by using both reports of thyroid disease and use of thyroid medications as indicative of maternal thyroid disease, we believe that the effects of recall bias were minimized. Another limitation was that infants with craniosynostosis likely received a variable work-up in the different study sites; true case infants could have been excluded because of the lack of radiographic confir- mation, and some case infants with a genetic cause might not have been recognized, and thus inappropriately included. Finally, it is difficult to disentangle effects of medications used to treat thyroid disease from those of the underlying disease, given the overlap between report of exposure to thyroid medication and of thyroid disease. However, the most commonly used medication reported by case mothers was thyroid hormone, known to cross the placenta only in small amounts. ${ }^{9}$

Our study also has several strengths. Although several case reports had previously suggested an association between maternal Graves' disease and craniosynostosis, data from this case-control study, which included an appropriate comparison group, provides stronger evidence for this association. Infants with craniosynostosis were ascertained through population-based birth defects surveillance systems at eight U.S. sites, with control infants randomly selected from the same population. The possibility of misdiagnosis, reported in some previous studies of craniosynostosis, ${ }^{10}$ was minimized because of the requirement for verification by radiographic imaging. Abstracted information on case infants was reviewed by a clinical geneticist, and those with recognized or strongly suspected single-gene disorders and chromosome abnormalities were excluded to reduce heterogeneity of the case group. ${ }^{27}$ Finally, although the number of case infants was relatively small, our study was large enough to allow consideration of several potential confounding factors.

Our study, which suggests an association between maternal thyroid disease (most likely Graves' disease) or its treatment and craniosynostosis in the infant, highlights the fact that thyroid function in the mother might not always accurately reflect the risk to the fetus, largely due to the presence of TSIs. These results emphasize the importance of identification of women with active or a previous history of thyroid disease, so that appropriate monitoring and optimal treatment can be provided.

\section{REFERENCES}

1. American College of Obstetricians and Gynecologists. ACOG Practice Bulletin. Clinical management guidelines for obstetrician-gynecologists. Number 37, August 2002. (Replaces Practice Bulletin Number 32, November 2001). Thyroid disease in pregnancy. Obstet Gynecol 2002;100:387-96.

2. Power ML, Kilpatrick S, Schulkin J. Diagnosing and managing thyroid disorders during pregnancy: a survey of obstetriciangynecologists. J Reprod Med 2004;49:79-82.

3. Smallridge RC, Glinoer D, Hollowell JG, Brent G. Thyroid function inside and outside of pregnancy: what do we know and what don't we know? Thyroid 2005;15:54-9. 
4. Casey BM, Leveno KJ. Thyroid disease in pregnancy. Obstet Gynecol 2006;108:1283-92.

5. Lazarus JH. Epidemiology and prevention of thyroid disease in pregnancy [published erratum appears in Thyroid 2003;13: 415]. Thyroid 2002;12:861-5.

6. Haddow JE, Palomaki GE, Allan WC, Williams JR, Knight GJ, Gagnon J, et al. Maternal thyroid deficiency during pregnancy and subsequent neuropsychological development of the child. N Engl J Med 1999;341:549-55.

7. Pop VJ, Brouwers EP, Vader HL, Vulsma T, van Baar AL, de Vijlder JJ. Maternal hypothyroxinaemia during early pregnancy and subsequent child development: a 3-year follow-up study. Clin Endocrinol (Oxf) 2003;59:282-8.

8. Lazarus JH. Thyroid disorders associated with pregnancy: etiology, diagnosis, and management. Treat Endocrinol 2005; 4:31-41.

9. Laurberg P, Nygaard B, Glinoer D, Grussendorf M, Orgiazzi J. Guidelines for TSH-receptor antibody measurements in pregnancy: results of an evidence-based symposium organized by the European Thyroid Association. Eur J Endocrinol 1998; 139:584-6.

10. Singer S, Bower C, Southall P, Goldblatt J. Craniosynostosis in Western Australia, 1980-1994: a population-based study. Am J Med Genet 1999;83:382-7.

11. Cohen MM Jr, Editorial: perspectives on craniosynostosis. Am J Med Genet A 2005;136:313-26.

12. Jehee FS, Johnson D, Alonso LG, Cavalcanti DP, de Sa Moreira E, Alberto FL, et al. Molecular screening for microdeletions at 9p22-p24 and 11q23-q24 in a large cohort of patients with trigonocephaly. Clin Genet 2005;67:503-10.

13. Robinson DC, Hall R, Munro DS. Graves's disease, an unusual complication: raised intracranial pressure due to premature fusion of skull sutures. Arch Dis Child 1969;44:252-7.

14. Menking M, Wiebel J, Schmid WU, Schmidt WT, Ebel KD, Ritter R. Premature craniosynostosis associated with hyperthyroidism in 4 children with reference to 5 further cases in the literature. Monatsschr Kinderheilkd 1972;120:106-10.

15. Duggan CA, Keener EB, Gay BB. Secondary craniosynostosis. AJR Am J Roentgenol 1970;109:277-293.

16. Biebermann $H$, Schoneberg $T$, Krude $H$, Gudermann $T$, Gruters A. Constitutively activating TSH-receptor mutations as a molecular cause of non-autoimmune hyperthyroidism in childhood. Langenbecks Arch Surg 2000;385:390-2.

17. Segni M, Leonardi E, Mazzoncini B, Pucarelli I, Pasquino AM. Special features of Graves' disease in early childhood. Thyroid 1999;9:871-7.

18. Wilroy RS Jr, Etteldorf JN. Familial hyperthyroidism including two siblings with neonatal Graves' disease. J Pediatr 1971;78: 625-32.

19. Johnsonbaugh RE, Bryan RN, Hierlwimmer R, Georges LP. Premature craniosynostosis: a common complication of juvenile thyrotoxicosis. J Pediatr 1978;93:188-91.

20. Penfold JL, Simpson DA. Premature craniosynostosis-a complication of thyroid replacement therapy. J Pediatr 1975;86: 360-3.

21. Daneman D, Howard NJ. Neonatal thyrotoxicosis: intellectual impairment and craniosynostosis in later years. J Pediatr 1980;97:257-9.

22. Cove DH, Johnston P. Fetal hyperthyroidism: experience of treatment in four siblings. Lancet 1985;1:430-2.

23. de Lima MA, Oliveira LB, Paim N, Borges Mde F. Congenital hyperthyroidism: autopsy report. Rev Hosp Clin Fac Med Sao Paulo 1999;54:103-6.
24. Krude H, Biebermann H, Krohn HP, Dralle H, Gruters A. Congenital hyperthyroidism. Exp Clin Endocrinol Diabetes 1997;105 suppl:6-11.

25. Stevenson RE, Trent HE. Maternal hyperthyroidism and congenital craniosynostosis. Proc Greenwood Genet Center 1990;9:3-6.

26. Yoon PW, Rasmussen SA, Lynberg MC, Moore CA, Anderka M, Carmichael SL, et al The National Birth Defects Prevention Study. Public Health Rep 2001;116 suppl:32-40.

27. Rasmussen SA, Olney RS, Holmes LB, Lin AE, KepplerNoreuil KM, Moore CA, et al. Guidelines for case classification for the National Birth Defects Prevention Study. Birth Defects Res A Clin Mol Teratol 2003;67:193-201.

28. Harvey CB, O'Shea PJ, Scott AJ, Robson H, Siebler T, Shalet $\mathrm{SM}$, et al. Molecular mechanisms of thyroid hormone effects on bone growth and function. Mol Genet Metab 2002;75: $17-30$.

29. Akita S, Nakamura T, Hirano A, Fujii T, Yamashita S. Thyroid hormone action on rat calvarial sutures. Thyroid 1994;4: 99-106.

30. LaFranchi S. Congenital hypothyroidism: etiologies, diagnosis, and management. Thyroid 1999;9:735-40.

31. Momotani N, Ito K, Hamada N, Ban Y, Nishikawa Y, Mimura T. Maternal hyperthyroidism and congenital malformation in the offspring. Clin Endocrinol (Oxf) 1984;20:695-700.

32. Khoury MJ, Becerra JE, d'Almada PJ. Maternal thyroid disease and risk of birth defects in offspring: a population-based case-control study. Paediatr Perinat Epidemiol 1989;3: 402-20.

33. Wing DA, Millar LK, Koonings PP, Montoro MN, Mestman $\mathrm{JH}$. A comparison of propylthiouracil versus methimazole in the treatment of hyperthyroidism in pregnancy. Am J Obstet Gynecol 1994;170:90-5.

34. Gardner JS, Guyard-Boileau B, Alderman BW, Fernbach SK, Greene C, Mangione EJ. Maternal exposure to prescription and non-prescription pharmaceuticals or drugs of abuse and risk of craniosynostosis. Int J Epidemiol 1998;27:64-7.

35. Ogilvy-Stuart AL. Neonatal thyroid disorders. Arch Dis Child Fetal Neonatal Ed 2002;87:F165-71.

36. Hoffman WH, Sahasrananan P, Ferandos SS, Burek CL, Rose NR. Transient thyrotoxicosis in an infant delivered to a long-acting thyroid stimulator (LATS)- and LATS protectornegative, thyroid-stimulating antibody-positive woman with Hashimoto's thyroiditis. J Clin Endocrinol Metab 1982;54: $354-6$.

37. Zakarija M, McKenzie JM. Pregnancy-associated changes in the thyroid-stimulating antibody of Graves' disease and the relationship to neonatal hyperthyroidism. J Clin Endocrinol Metab 1983;57:1036-40.

38. Kohn LD, Suzuki K, Hoffman WH, Tombaccini D, Marcocci $\mathrm{C}$, Shimojo N, et al. Characterization of monoclonal thyroidstimulating and thyrotropin binding-inhibiting autoantibodies from a Hashimoto's patient whose children had intrauterine and neonatal thyroid disease. J Clin Endocrinol Metab 1997; 82:3998-4009.

39. Peleg D, Cada S, Peleg A, Ben-Ami M. The relationship between maternal serum thyroid-stimulating immunoglobulin and fetal and neonatal thyrotoxicosis. Obstet Gynecol 2002; 99:1040-3.

40. Dirmikis SM, Munro DS. Placental transmission of thyroidstimulating immunoglobulins. Br Med J 1975;2:665-6. 
41. Kilpatrick S. Umbilical blood sampling in women with thyroid disease in pregnancy: is it necessary? Am J Obstet Gynecol 2003; 189:1-2.

42. Luton D, Le Gac I, Vuillard E, Castanet M, Guibourdenche J, Noel M, et al. Management of Graves' disease during pregnancy: the key role of fetal thyroid gland monitoring. J Clin Endocrinol Metab 2005;90:6093-8.
43. Nachum Z, Rakover Y, Weiner E, Shalev E. Graves' disease in pregnancy: prospective evaluation of a selective invasive treatment protocol. Am J Obstet Gynecol 2003;189: 159-65.

44. McNab T, Ginsberg J. Use of anti-thyroid drugs in euthyroid pregnant women with previous Graves' disease. Clin Invest Med 2005;28:127-31.

\section{OBSTETRICS\&}

GYNECOLOGY

\section{Submitting a Clinical Trial? Register Your Trial in a Public Trials Registry}

All clinical trials submitted to Obstetrics \& Gynecology must be registered in a public trials registry at or before the onset of patient enrollment. ${ }^{1-3}$ The International Committee of Medical Journal Editors (ICMJE) defines a clinical trial as "any research project that prospectively assigns human subjects to intervention or comparison groups to study the cause-and-effect relationship between a medical intervention and a health outcome. Studies designed for other purposes, such as to study pharmacokinetics or major toxicity (eg, phase I trials), would be exempt."1

Registries approved by the International Committee of Medical Journal Editors are: ${ }^{4}$

- www.clinicaltrials.gov

- isrctn.org

- www.umin.ac.jp/ctr/index.htm

- www.actr.org.au

- www.trialregister.nl/trialreg/index.asp

Authors should provide the name of the trial registry, the registry URL, and the trial registration number at the end of the abstract.

\section{References}

1. International Committee of Medical Journal Editors. Uniform requirements for manuscripts submitted to biomedical journals. Available at: http://www.icmje.org. Retrieved September 15, 2005.

2. DeAngelis GD, Drazen JM, Frizelle FA, Haug G, Hoey J, Horton R, et al. Clinical trial registration: a statement from the International Committee of Medical Journal Editors. JAMA 2004;292:1363-4.

3. DeAngelis CD, Drazen JM, Frizelle FA, Haug C, Hoey J, Horton R, et al. Is this clinical trial fully registered?: a statement from the International Committee of Medical Journal Editors. JAMA 2005;293:2927-9.

4. Council of Science Editors. CSE endorsement of principles: ICMJE's statement on clinical trial registration. Available at: http://www.councilscienceeditors.org/editorial_policies/endorsementofprinciples.cfm. Retrieved January 16, 2007. 